\title{
DEFORMATIONS OF GROUP ACTIONS
}

\author{
DAVID FISHER
}

\begin{abstract}
Let $G$ be a non-compact real algebraic group and $\Gamma<G$ a lattice. One purpose of this paper is to show that there is a smooth, volume preserving, mixing action of $G$ or $\Gamma$ on a compact manifold which admits a smooth deformation. In fact, we prove a stronger statement by exhibiting large finite dimensional spaces of deformations. We also describe some other, rather special, deformations when $G=S O(1, n)$.
\end{abstract}

\section{INTRODUCTION}

In recent years, many results have been proven concerning local rigidity of group actions. Most known results concern actions of semi-simple Lie groups with all simple factors of real rank at least two and lattices in such groups. For the best known results in that category and some historical references, see [6]. More recently some partial results have been proven for more general groups, including lattices in $S p(1, n)$ and $F_{4}^{-20}$; see [7] and [11]. In this context it is interesting to ask when Lie groups and their lattices have actions by diffeomorphisms which admit nontrivial perturbations or deformations. Actions of $S L(n, \mathbb{Z})$ constructed by Katok and Lewis admit non-trivial deformations and their construction was modified by Benveniste to produce actions of some higher rank simple Lie groups with nontrivial volume preserving deformations $[2,13]$. Benveniste's deformations are also non-trivial when all actions in question are restricted to any lattice in the acting group. Throughout this paper, by a connected real algebraic group, I mean the connected component of the real points of an algebraic group defined over $\mathbb{R}$. One of the main results of this paper is the following generalization of Benveniste's theorem:

Theorem 1.1. Let $G$ be a non-compact, simple, connected real algebraic group and $\Gamma<G$ a lattice. Then there is a smooth, volume preserving, mixing, action of $G$ which admits non-trivial, volume preserving deformations. Furthermore, the deformations remain non-trivial when restricted to $\Gamma$.

Remarks. (1) It is immediate from the construction that we can construct actions where the space of deformations has arbitrarily large, finite, dimension. It would be interesting to know when the deformation space is infinite dimensional. For any group $G$ which contains a lattice with a homomorphism onto $\mathbb{Z}$, it is easy to build examples with infinite dimensional families of perturbation using simple induction constructions and Lemma 2.3 below.

Received by the editors July 5, 2006.

2000 Mathematics Subject Classification. Primary 37C85.

The author was partially supported by NSF grant DMS-0226121 and a PSC-CUNY grant.

(C)2007 American Mathematical Society Reverts to public domain 28 years from publication 
(2) The construction of the actions and deformations is a refinement of Benveniste's which is based on an earlier construction of Katok and Lewis $[2,13]$. The proof that the deformations are non-trivial is new and uses a consequence of Ratner's theorem due to N. Shah. Benveniste's proof used commutativity coming from a higher rank assumption on $G$.

(3) Benveniste's explicit deformations are non-trivial in a sense that is weaker than what is meant by Theorem 1.1; see below for discussion.

We now introduce some basic notions in order to describe in detail the meaning of the statement of Theorem 1.1 and also to clarify Remark (1) and show that we produce "many" deformations. Let $\rho$ be an action of a group $D$ on a manifold $M$. Then the space of actions of $D$ on $M$ is naturally the space $\operatorname{Hom}(D, \operatorname{Diff}(M))$. The group $\operatorname{Diff}(M)$ acts on this space by conjugation, and two group actions are conjugate if and only if they are in the same $\operatorname{Diff}(M)$ orbit. Note that if $D$ is finitely generated, $\operatorname{Hom}(D, \operatorname{Diff}(M))$ is naturally a closed subset of the product of a finite number of copies of $\operatorname{Diff}(M)$. In the finite dimensional setting, i.e. where we replace $\operatorname{Diff}(M)$ by an algebraic group $H$, the space $\operatorname{Hom}(D, H)$ is an algebraic variety, and there is a construction of a quotient scheme $\operatorname{Hom}(D, H) / / H$ which allows one to study the space of non-trivial deformations. In the infinite dimensional setting it is unclear that there is any meaningful algebraic geometry, or that $\operatorname{Hom}(D, \operatorname{Diff}(M))$ is a manifold at "most" (or even any) points. We now describe in more detail the space of deformations produced in the proof of Theorem 1.1. We do this for $\Gamma$ actions, where the space $\operatorname{Hom}(\Gamma, \operatorname{Diff}(M))$ is at least a closed subset of a comprehensible topological space, the $k$-fold product $\operatorname{Diff}(M) \times \cdots \times \operatorname{Diff}(M)$ where $k$ is the number of generators of $\Gamma$. (There are various ways in which one can topologize $\operatorname{Hom}(G$, $\operatorname{Diff}(M))$, but this is more complicated, and we will not discuss it here.) Our construction produces an action of $\Gamma$ on a manifold $M$ and a collection of deformations which provide an embedding of a small, finite dimensional ball in $\operatorname{Hom}(\Gamma, \operatorname{Diff}(M))$ which intersects the $\operatorname{Diff}(M)$ orbit of $\rho$ only at $\rho$. Benveniste's construction, even using our proof in place of his, only produces an action $\rho$ with deformations which define a map of a ball into $\operatorname{Hom}(\Gamma$, Diff $(M))$ which intersects the $\operatorname{Diff}(M)$ orbit of $\rho$ at a countable set which could, a priori, accumulate on $\rho$. Since we do not know, in this context, that the representation variety is even locally a manifold, it is unclear that the two statements are equivalent. As will be clear below, given $\Gamma$ and a positive integer $n$ we can construct a manifold $M$ and an action $\rho$ such that the ball discussed above has dimension $n$. By "blowing up and gluing" along many distinct pairs of closed orbits, one can even arrange that $n$ is much larger than $\operatorname{dim}(M)$.

In addition to removing the rank restriction in Benveniste's theorem, our use of Ratner theory is sufficiently robust to allow us to use slight modifications of the examples for Theorem 1.1 to prove the following stronger theorem:

Theorem 1.2. Let $G$ be a connected, non-compact real algebraic group and $\Gamma<G$ a lattice. Then there is a faithful, smooth, volume preserving, ergodic, action of $G$ which admits non-trivial, volume preserving deformations. Furthermore, the deformations remain non-trivial when restricted to $\Gamma$.

Remarks. (1) As will be clear in the proof, Theorem 1.2 is only really difficult if the abelianization of $G$ is compact. See Proposition 2.2. 
(2) Theorem 1.2 is in a sense sharp, since any smooth action of a compact group is locally rigid by a theorem of Palais [17].

(3) Though the author knows of no claims in the literature, it is quite likely that one can prove local rigidity theorems for groups which are not semisimple. In particular, the results of [6] seem likely to hold for $G$ any algebraic group all of whose non-trivial factors are semisimple groups with all simple factors of real rank at least two and for lattices $\Gamma$ in such $G$. Any such $G$ or $\Gamma$ can be shown to have property $(T)$, so the arguments in [7] go through verbatim. The main difficulty in proving local rigidity for quasi-affine actions appears to be in generalizing some of the results of [8]. The results in [22] should be relevant to this generalization.

With the exception of some of the results in $[3,6,7]$, all local rigidity theorems known to the author are for actions on manifolds which are homogeneous. The deformations constructed to prove Theorems 1.1 and 1.2 above are on manifolds which are not homogeneous. In section 5 we show that some affine actions of lattices in $S O(1, n)$ on homogeneous manifolds have non-trivial deformations. This then implies that the same is true for some affine actions of $S O(1, n)$ as well.

One motivation for this paper is the so-called Zimmer program to classify actions of higher rank lattices; see [24, 25] for more details. In the context of both this program and other older rigidity results, it is not surprising that actions of rank 1 lattices exhibit greater flexibility than higher rank lattices.

\section{Preliminaries}

2.1. Invariant measures and centralizers of group actions. In this section we recall a consequence of work of Ratner and Shah on invariant measures which is instrumental in our proofs. For the proofs of Theorems 1.1 and 1.2, our use of this result will be based on the fact that the perturbations we construct are conjugate back to the original action on a set of full measure. Other applications in section 5 will be deformations that are trivial by construction on a "large" subgroup of the acting group. We let $\operatorname{Aff}(H / \Lambda)$ denote the affine group of $H / \Lambda$. This group consists of diffeomorphisms of $H / \Lambda$ which are covered by maps of the form $A \circ L_{h}$ where $A$ is an automorphism of $H$ such that $A(\Lambda)=\Lambda$ and $L_{h}$ is left translation by $H$. The group $\operatorname{Aff}(H / \Lambda)$ is a Lie group and is, in fact a quotient of a subgroup of $\operatorname{Aut}(H) \ltimes H$; see [8] for more discussion.

Corollary 2.1. Let $H$ be a connected real algebraic group, $\Lambda<H$ a lattice, $\mu_{H}$ the measure on $H / \Lambda$ induced by a fixed Haar measure on $H$ and $F<H$ a group that either

(1) contains a subgroup $F^{\prime}<F$ generated by unipotent elements,

(2) contains a subgroup $F^{\prime}<F$ such that $F^{\prime}$ is a lattice in a subgroup $F^{\prime \prime}<H$ generated by unipotent elements.

Further assume that $F^{\prime}$ acts ergodically on $H / \Lambda$. If $\phi$ is an essentially surjective, essentially injective measurable map from $H / \Lambda$ to $H / \Lambda$ which commutes with the $F$ action, then $\phi$ is a translation by an element of the centralizer of $F^{\prime}$ in $\operatorname{Aff}(H / \Lambda)$.

This corollary is essentially noted in Witte's paper [21] where a more general result is proven. For completeness, we briefly recall the proof. 
Proof. If $\phi$ commutes with $F^{\prime}$, then the graph $N$ of $\phi$ is preserved by $F^{\prime}$. Letting $\tilde{\phi}: H / \Lambda \rightarrow H / \Lambda \times H / \Lambda$ be given by $\tilde{\phi}=(\mathrm{Id}, \phi)$, it is straightforward to check that $\tilde{\phi}_{*} \mu_{H}$ is an $F^{\prime}$ invariant, ergodic measure on $N$ that projects to $\mu_{H}$ on each $H / \Lambda$. Given a subgroup $L<H$, we denote by $\Delta(L)$ the diagonal embedding of $L$ in $H \times H$. By Shah's extensions of Ratner's theorem to groups generated by unipotents and their lattices [19, 20], this implies that $N$ is a closed $L$ orbit in $H / \Lambda \times H / \Lambda$ where $L$ is a closed subgroup of $H \times H$ containing $\Delta\left(F^{\prime}\right)$. Since $\tilde{\phi}$ composed with projection on the second factor is essentially injective and essentially surjective, we have that $L$ is exactly $\Delta(H)$. This implies that $N$ is a closed $\Delta(H)$ orbit in $H / \Lambda \times H / \Lambda$, which implies that $\phi$ is a translation by an element of $H$ composed with an automorphism of $H$. This implies that $\phi$ is in fact an element of $Z_{\mathrm{Aff}(H / \Lambda)}\left(F^{\prime}\right)$.

2.2. Reductions for the proof of Theorem 1.2. We briefly recall some facts concerning (real) algebraic groups. Given a connected real algebraic group $G$, we can write $G$ as a semi-direct product $L \ltimes U$ where $U, L<G$ and $U$ is the unipotent radical of $G$ and $L$ is a reductive real algebraic group which is called a Levi complement.

Proposition 2.2. To prove Theorem 1.2, it suffices to consider the case when the Levi complement of $G$ has compact center.

Proof. We assume that the center of the Levi complement is non-compact and produce the family of perturbations required in Theorem 1.2. We have a projection $\pi_{1}: G \rightarrow L$, so any $L$ action defines a $G$ action. We can write $L$ as $S Z(L)$ where $S$ is semisimple, $S \cap Z(L)=F$ is finite and the product is almost direct. Therefore $S$ is normal in $L$ and there is a projection $\pi_{2}: L \rightarrow Z(L) / F$. The group $Z(L) / F$ is an abelian Lie group and non-compact whenever $Z(L)$ is non-compact, so we can find a third projection $\pi_{3}: Z(L) / F \rightarrow \mathbb{R}$. We write $\pi=\pi_{3} \circ \pi_{2} \circ \pi_{1}$ and note that any $\mathbb{R}$ action defines a $G$ action via composition with $\pi$.

There are many $\mathbb{R}$ actions which are smooth and mixing and which have nontrivial perturbations; we give an example for completeness. Let $\Lambda<S L(2, \mathbb{R})$ be a cocompact lattice and let $\rho_{\mathbb{R}}$ be the action of $\mathbb{R}$ on $S L(2, \mathbb{R}) / \Lambda$ defined by the horocycle flow. (I.e. defined by identifying $\mathbb{R}$ with strictly upper triangular matrices in $S L(2, \mathbb{R})$ and acting by left translation.) It is easy to perturb this action, even as an action by left translations, since this action has zero entropy and many nearby actions defined by "nearby" subgroups of $S L(2, \mathbb{R})$ have positive entropy. It is straightforward to construct families on which the entropy is strictly increasing as one moves away from $\rho_{\mathbb{R}}$. Since Lyapunov exponents are also conjugacy invariants of diffeomorphisms, by choosing an $\mathbb{R}$ action on a larger manifold, one can construct large dimensional families of deformations which are easily seen to be non-trivial and to give rise to non-trivial deformations of the faithful actions described in the next paragraph.

To construct a faithful action of $G$ with pertubations, we embed $G$ in $S L(N+1, \mathbb{R})$, choose a cocompact lattice $\Delta<S L(N+1, \mathbb{R})$ and let $\rho_{F}$ be the left translation action of $G$ on $S L(N+1, \mathbb{R}) / \Delta$. We then form

$$
M=S L(2, \mathbb{R}) / \Lambda \times S L(N+1, \mathbb{R}) / \Delta
$$

and let $\rho$ be the diagonal $G$ action defined by $\pi \circ \rho_{\mathbb{R}}$ and $\rho_{F}$. It is straightforward to check that this satisfies the hypotheses of Theorem 1.2. The fact that the action 
is mixing follows from the Howe-Moore theorem; see e.g. [23]. It is also straightforward to check that $\left.\rho\right|_{\Gamma}$ provides the necessary action of $\Gamma$ when $\Gamma<G$ is a lattice.

We now turn to the case where the entire Levi complement is compact. In order to prove the existence of deformations in this case, we will need to use induced actions. Let $F$ be a locally compact topological group and $D<F$ a closed subgroup. Assume $D$ acts continuously on a compact space $X$. We can then form the space $(F \times X) / D$ where $D$ acts by $(f, x) d=\left(f d^{-1}, d x\right)$. This space is clearly equipped with a left $F$ action which we refer to as the induced $F$ action. It is easy to check that if $F$ is a Lie group, $X$ is a smooth manifold and $D$ acts smoothly on $X$, then the induced $F$ action is also smooth.

Lemma 2.3. If $H$ is a real Lie group and $L<H$ is a closed cocompact subgroup, then if $L$ has an action on a compact manifold $M$ which admits non-trivial deformations (resp. perturbations), the induced $H$ action on $(H \times M) / L$ also admits non-trivial deformations (resp. perturbations).

Proof. Let $\rho_{0}$ be an $L$ action on $M$ and $\rho_{t}$ a non-trivial one parameter deformation. We can form the induced actions $\tilde{\rho}_{t}$ of $H$ on $(H \times M) / L$ which are again smooth actions and it is clear that $\tilde{\rho}_{t}$ is a one parameter deformation of $\tilde{\rho}_{0}$. For a contradiction, assume that deformations $\tilde{\rho}_{t}$ are trivial. Then there exist diffeomorphisms $\phi_{t}$ of $(H \times M) / L$ such that $\phi_{t} \circ \rho_{0}=\rho_{t} \circ \phi_{t}$. Let $\pi:(H \times M) / L \rightarrow H / L$ be the natural projection. Then $\phi_{t} \circ \pi$ is an $H$ equivariant map from $\left((H \times M) / L, \rho_{t}\right)$ to $\left(H / L, \rho_{H}\right)$ which is close to $\pi$ as a smooth map. The sets $\left(\phi_{t} \circ \pi\right)^{-1}([h])$ are just the sets $\phi_{t}^{-1}\left(\left(\pi^{-1}[h]\right)\right)$ and so are an $H$ invariant foliation of $(H \times M) / L$. In particular $\phi_{t}^{-1}\left(\left(\pi^{-1}[e]\right)\right)$ is an $L$ invariant copy of $M$, and it is easy to check that the $L$ action on $\phi_{t}^{-1}\left(\pi^{-1}[e]\right)$ is exactly $\rho_{t}$. Therefore $\left.\phi_{t}\right|_{\pi^{-1}([e])}$ is a conjugacy between $\rho_{0}$ and $\rho_{t}$. It is straightforward to check that $\left.\phi_{t}\right|_{\pi^{-1}([e])}$ is indeed a small, smooth path in $\operatorname{Diff}(M)$. The same proof applies to perturbations in place of deformations.

Proposition 2.4. It suffices to prove Theorem 1.2 when the Levi complement of $G$ is not compact.

Proof. We prove that if the Levi complement is compact, then there are deformations. By this assumption, $G=C \ltimes U$ where $C$ is compact and $U$ is unipotent. There exist non-trivial homomorphisms $\sigma: U \rightarrow \mathbb{R}$. As in the proof of Proposition 2.2 , we can use an $\mathbb{R}$ action $\rho_{\mathbb{R}}$ with non-trivial perturbations to construct a $U$ action with non-trivial perturbations. This yields a $G$ action with non-trivial perturbations by Lemma 2.3. The $G$ action is volume preserving if $\rho_{\mathbb{R}}$ is, since there is a $G$ invariant volume on $G / U$. It is easy to check that this action is mixing when $\rho_{\mathbb{R}}$ is mixing. To obtain a faithful, mixing action, one proceeds as in Proposition 2.2. To verify that the deformations are non-trivial on the faithful action requires some care, and we sketch a simple argument using entropy or Lyapunov exponents. Let $B<U$ be a one parameter subgroup mapped onto $\mathbb{R}$ by $\sigma$. Then the restriction of the induced action to $B$ is the product of the trivial $B$ action on $G / U$ with the action $\left.\rho_{\mathbb{R}} \circ \sigma\right|_{B}$. This shows that the Lyapunov exponents or entropy of $\rho_{\mathbb{R}}$ are an invariant of the induced action, and therefore of the diagonal action used to define a faithful $G$ action. 
Given $\Gamma<G$, a theorem of Auslander shows that $\Gamma \cap U$ is a lattice in $U$ and therefore that $\sigma(\Gamma \cap U)$ is infinite and unbounded [1]. From this it is easy to check that the restriction of the above action to $\Gamma$ proves Theorem 1.2 for $\Gamma$ actions.

Remarks. (1) The techniques of this subsection are really only necessary for the case of a non-compact real algebraic group all of whose simple quotients are compact. As long as there is a non-compact simple quotient, one can proceed by the methods of section 4 .

(2) Here we only use rough dynamical invariants such as entropy and Lyapunov exponents to see that perturbations are non-trivial. Using finer dynamical invariants, one can easily see that the spaces of deformations yielded by Proposition 2.2 and Proposition 2.4 are infinite dimensional. We do not deal with this here, since it would lead to a long digression on classical dynamics.

\section{Constructing "EXotic aCtions"}

We work with a variant of a construction developed by Benveniste in [2], which is inspired by the construction in [13]. Let $H=S L(n, \mathbb{R})$ and $J=S L(m, \mathbb{R})$ with $m<n-1$. View $J<H$ as a subgroup via the standard embedding as block $m$ by $m$ matrices in the upper left corner. For applications we will choose $m$ large enough so that $G<S L(m, \mathbb{R})=J$. We let $Z=S L(n-m, \mathbb{R})<Z_{H}(J)$ be the block $n-m$ by $n-m$ matrices in the lower right hand corner. In fact $Z_{H}(J)=Z(H) D Z$ where $Z(H)$ is the center of $H$ and $D$ is the group of diagonal matrices in $H$ commuting with $J \times Z$, i.e. the group of diagonal matrices of the form $\operatorname{diag}\left\{d_{1}, \ldots, d_{m}, d_{m+1}, \ldots, d_{n}\right\}$ with $d_{1}=d_{2}=\cdots=d_{m}$ and $d_{m+1}=\cdots=d_{n}$. Note that $D<H$ forces $d_{1}^{m} d_{n+1}^{n-m}=1$. We recall the existence of certain types of lattices in $H$.

Lemma 3.1. If $n-m$ is a multiple of $m$, there exists a cocompact lattice $\Lambda<H$ such that:

(1) $\Lambda \cap J=\Lambda_{J}$ is a cocompact lattice in $J$,

(2) $\Lambda \cap Z=\Lambda_{Z}$ is a cocompact lattice in $Z$,

(3) $\Lambda \cap D$ is trivial,

(4) $\Lambda \cap D J Z=\Lambda_{J} \times \Lambda_{Z}$

Proof. This follows easily from general methods to construct cocompact lattices using unitary groups of Hermitian forms. See for example [16, Proposition 7.54 and Figure 10.2] for a construction that easily yields lattices where the first two items are clear. Since $D$ is diagonalizable over $\mathbb{Q}$ (i.e. $\mathbb{Q}$ isotropic), point (3) follows from [18, Theorem 4.11]. Point 4 follows from [18, Theorem 4.13] or rather from part of its proof and the use of change of base to realize $\Lambda$ as the integer points for a $\mathbb{Q}$-structure on $H$ that restricts to a $\mathbb{Q}$-structure on $D J Z$.

Note that $J Z=S L(m, \mathbb{R}) \times S L(n-m, \mathbb{R})<S L(n, \mathbb{R})$ is a closed subgroup such that $J Z \cap \Lambda=\Lambda_{J Z}$ is a lattice in $J Z$. To simplify some arguments, we pass to a subgroup of finite index in $\Lambda$ to guarantee that $\Lambda$ does not intersect the center of $J, H$ or $Z$. Let $M=H / \Lambda$. If $h \in H$, we will write [h] for the image of $h$ in $M$. The following is now easy from our choices of groups, subgroups and lattice. 
Corollary 3.2. The orbit $Z(H) J Z D[e]$ is one-to-one immersed. The orbit $J[e]$ is an embedded submanifold $J$ equivariantly diffeomorphic to $J / \Lambda_{J}$ and the orbit $J Z[e]$ is an embedded submanifold which is $J Z$ equivariantly diffeomorphic to $J / \Lambda_{J} \times Z / \Lambda_{Z}$. In particular, if $z \in Z_{H}(J)$ and $z J[e] \subset J Z[e]$, then $z \in Z$.

Proof. It is a standard fact that the orbit of a point $x$ in a homogeneous space for a Lie group $H$ under a closed subgroup $L$ is an immersed submanifold which is equivariantly diffeomorphic to $L / L_{x}$ where $L_{x}$ is the stabilizer of $x$ in $L$. Furthermore, the orbit is embedded if $L / L_{x}$ is compact. Combined with Lemma 3.1, this yields the corollary immediately.

Remark. The construction in [2] involves less careful choices of subgroups and lattices, and this care is taken here to allow us to show the existence of an embedded disk in the space of deformations.

Note that for $z \in Z$ small enough, $z J[e]$ does not intersect the closed set $J[e]$. Let $C_{0} \subset H / \Lambda$ be the orbit $J[e]$ and $C_{1} \subset H / \Lambda$ be the orbit $J[z]$, where the action of $J$ is the obvious left action on $H / \Lambda$; the sets $C_{0}$ and $C_{1}$ are closed submanifolds (by our assumption on $\Lambda$ ) and are disjoint. We now form a manifold $Y_{z}$ by blowing up $C_{0}$ and $C_{1}$ and gluing the resulting exceptional divisors. More precisely, define $Y_{z}$ as follows.

We may identify $T M$ with $M \times \mathfrak{h}$, and then the derivative action of $H$ on $T M$ is given by $h(m, X)=(h m, A d(h) X)$. The tangent bundle to the $J$-orbits is then clearly $M \times \mathfrak{j}$; since the adjoint action of $J$ on $\mathfrak{h}$ is reductive, there is a $J$-invariant complement $V$ to $\mathfrak{j}$, and $M \times V$ is a $J$ - invariant subbundle of $T M$ which restricts to a normal bundle for $C_{0}$ or $C_{1}$. Define maps

$$
\phi_{i}: J \times_{\Lambda_{J}} V \rightarrow M, i=0,1,
$$

by

$$
\phi_{0}([j], v)=j \exp (v)[e], \phi_{1}(h, v)=j \exp (v[z] .
$$

These maps are clearly well-defined and $J$-equivariant, and there are open neighborhoods $U_{i} \subset J \times_{\Lambda_{J}} V$ of $J \times_{\Lambda_{J}} 0$ such that $\phi_{i}$ restricted to $U_{i}$ is a diffeomorphism onto a neighborhood of $C_{i}$, for $i=0,1$. Now let $S^{+}=(V-0) / \mathbb{R}^{+}$and let $L^{+} \rightarrow S^{+}$ be the tautological bundle of (closed) rays over $S^{+}$, i.e.

$$
L^{+}=\left\{(v,[w]) \in V \times S^{+} \mid v=r w, r \geq 0\right\} .
$$

Denote the zero-section of $L^{+}$by $S$. Also, let $L \rightarrow S^{+}$denote the tautological line bundle; observe that $L$ is two copies of $L^{+}$glued along $S$. The group $G L(V)$ acts on $L, L^{+}, S^{+}$, and $S$ and therefore $J$ does as well. We can then form the space $J \times_{\Lambda_{J}} L^{+}$, and there is a $J$-equivariant embedding $V-0 \rightarrow L^{+}$. Let $\hat{U}_{i}=$ $\left(U_{i}-\left(J \times{ }_{\Lambda_{J}} 0\right)\right) \cup S$, where $U_{i}$ is regarded as a subset of $J \times{ }_{\Lambda_{J}} L^{+}$through the above embedding for $i=0,1$. Now let

$$
M_{z}=\left(\left(M-\left(C_{0} \cup C_{1}\right)\right) \sqcup \hat{U}_{0} \sqcup \hat{U}_{1}\right) / \mathcal{R}
$$

where the equivalence relation $\mathcal{R}$ is generated by requiring $u \equiv \phi_{i}(u)$ for $u \in U_{i}$ and $\phi_{i}(u) \in M-\left(C_{0} \cup C_{1}\right) . M_{z}$ is then a manifold with boundary with two boundary components $B_{0}$ and $B_{1}$, each diffeomorphic to $J \times_{\Lambda_{J}} S^{+}$; it is also equipped with a $J$-action by declaring the action to be the restriction of the action on $M$ to $M_{z}-\left(B_{0} \cup B_{1}\right)=M-\left(C_{0} \cup C_{1}\right)$, and the obvious action on $B_{i}=J \times_{\Lambda_{J}} S^{+}$for $i=0,1$. This action is smooth by construction. 
Now let $Y_{z}$ be the manifold obtained by gluing $B_{0}$ to $B_{1}$ in the following way: identifying each of these boundary components to $J \times_{\Lambda_{J}} S^{+}$, glue the point $(j, s)$ on $B_{0}$ to the point $(j,-s)$ on $B_{1}$; this is obviously well-defined. It is obvious too that the action on $M_{z}$ descends to $Y_{z}$. Let $B_{z}$ be the image of the $B_{i}$ s in $Y_{z}$, and let $U$ be $Y_{z}-B=M-\left(C_{0} \cup C_{1}\right) ; B$ is a closed $J$-invariant set and $U$ is an open dense $J$-invariant set. Note that there is a $J$-map $\phi: J \times_{\Lambda_{J}} L \rightarrow Y_{z}$ and neighborhoods $W$ of $J \times_{\Lambda_{J}} S^{+}$in $J \times_{\Lambda_{J}} L$ and $W^{\prime}$ of $B$ in $Y_{z}$ such that $\left.\phi\right|_{W}: W \rightarrow W^{\prime}$ is a diffeomorphism.

The Haar measure on $H / \Lambda$ clearly determines a finite $J$-invariant measure $\mu$ on the spaces $Y_{z}$ described above; but these support no smooth invariant volume form. Indeed, suppose $\nu$ were such a volume form, and let $\frac{d \nu}{d \mu}$ be the RadonNikodym derivative of (the measure defined by) $\nu$ with respect to $\mu$. Then this is a $J$-invariant function and so, by ergodicity, constant almost everywhere; thus $\nu$ is a constant multiple of $\mu$. But an easy calculation shows that $\mu$ is (defined by) a smooth form on $Y_{z}$ which vanishes on the submanifold $B$, since $\nu$ must be a constant multiple of this form it is not a volume form.

However, imitating Katok-Lewis and Benveniste, we can modify the action near $B$ to create a volume-preserving one. Let

$$
\pi: J \times_{\Lambda_{J}} L \rightarrow J / \Lambda_{J}
$$

be the natural projection, and $d H$ be the Haar volume form on $H / \Lambda$. Then, considering $V-0$ as a subset of $L$, one easily finds that

$$
\phi^{*} \mu=\pi^{*} d H \wedge \Omega
$$

on $W \cap H \times_{\Lambda}(V-0)$ where $\Omega$ is a $J$ - invariant volume form on the vector space $V$. Now let $n$ be the dimension of $V$. There is a $G L(V)$-equivariant (non-linear) map $k: L \rightarrow L^{\otimes n}$ defined by $v \rightarrow \epsilon(v)^{(n+1)} v \otimes v \otimes \ldots \otimes v$, where $\epsilon(v)=1$ if $v$ has the same orientation as its image in $S^{+}$, and $=-1$ otherwise. Note that $L^{\otimes n}$ is a line bundle and $k$ is a diffeomorphism on the complement of the zero-section. Thus there is a map

$$
K: J \times_{\Lambda_{J}} L \rightarrow J \times_{\Lambda_{J}} L^{\otimes n}
$$

which is a diffeomorphism on the complement of $J \times_{\Lambda_{J}} S^{+}$. Let $Y_{z}^{\prime}$ be the manifold

$$
Y_{z}-B \sqcup W^{\prime \prime} / \equiv
$$

where $W^{\prime \prime}$ is a sufficiently small neigborhood of the zero - section in $J \times_{\Lambda_{J}} L^{\otimes n}$ and the relation $\equiv$ is generated by $w \equiv \phi \circ K^{-1}(w)$ for $w \in W^{\prime \prime}-J \times_{\Lambda_{J}} S^{+}$. Since the actions of $J$ correspond under this equivalence, there is a smooth action $\rho_{z}^{\prime}$ of $J$ on $Y_{z}^{\prime}$.

Note that $Y_{z}^{\prime}$ and $Y_{z}$ are easily seen to be diffeomorphic. It is also easy to see, using Ratner's theorem, that they are not $J$ equivariantly diffeomorphic, or even $F$ equivariantly diffeomorphic when $F$ is any subgroup containing a unipotent element. See [9, Section 4] for more discussion of these observations in the special case of the examples of Katok and Lewis; the arguments there carry over more or less verbatim to this case.

Now choose a positive definite inner product on $V$; this defines trivializations of $L$ and all associated bundles, and a volume $\Theta$ on $S^{+}$; in terms of these trivializations, 
the map $k$ above can be written

$$
\begin{gathered}
k: \mathbb{R} \times S^{+} \rightarrow \mathbb{R} \times S^{+}, \\
(r, l) \rightarrow\left(\operatorname{sgn}(r)^{n+1} r^{n}, l\right) .
\end{gathered}
$$

Since $\Omega=c r^{n-1} d r \wedge \Theta$, where $c$ is some constant, we have $\Omega=k^{*} c^{\prime} d r \wedge \Theta$ on the complement of the zero section, and so $K^{-1 *}\left(\pi^{*} d H \wedge \Omega\right)$ defines a form which extends to a non-zero volume form on all of $W^{\prime \prime}$. Thus the form $\Sigma$ on $Y_{z}^{\prime}$ defined by

$$
\begin{gathered}
\left.\Sigma\right|_{Y_{z}-B}=\mu, \\
\left.\Sigma\right|_{W^{\prime \prime}}=K^{-1 *}\left(\pi^{*} d H \wedge \Omega\right)
\end{gathered}
$$

defines a smooth volume on $Y_{z}^{\prime}$ which is obviously $J$ invariant. Thus

Proposition 3.3. The $J$ action $\rho^{\prime}$ on $Y_{z}^{\prime}$ preserves a smooth volume.

\section{Deformations of the exotic aCtions}

We now wish to study how the actions constructed above depend on the parameter $z$. We fix a norm on $\mathfrak{z}$. Let $z_{0} \in Z$. For $z \in Z$ close to $z_{0}, Y_{z}^{\prime}$ is diffeomorphic to $Y_{z_{0}}^{\prime}$, which we abbreviate as $Y$. We can then consider that as $z$ varies we obtain varying actions

$$
\rho_{z}^{\prime}: J \rightarrow \operatorname{Diff}(Y)
$$

by choosing the diffeomorphisms of $Y_{z}^{\prime}$ with $Y$ suitably; we can assume that $\rho_{z}^{\prime}$ varies differentiably in $z$. More explicitly, let $f$ be a function on $H / \Lambda$ that is equal to one on a small neighborhood of $H\left[z_{0}\right]$ and 0 outside a slightly larger neighborhood. Then for some $\varepsilon>0$ and any $v$ in $\mathfrak{z}$ with $\|v\|<\varepsilon$, the maps $\exp (f v)$ give a family of diffeomorphisms

$$
\phi_{v}: H / \Lambda-\left(J\left[z_{0}\right] \cup J[e]\right) \rightarrow H / \Lambda-\left(J\left[(\exp (f v)) z_{0}\right] \cup J[e]\right),\|v\|<\epsilon .
$$

These extend to diffeomorphisms

$$
\phi_{v}: Y_{z_{0}}^{\prime} \rightarrow Y_{(\exp (f v)) z_{0}}^{\prime}
$$

such that if

$$
\alpha_{z}: J \rightarrow \operatorname{Diff}\left(Y_{z}^{\prime}\right)
$$

are the $J$-actions defined above, we can define $\rho_{v}: J \rightarrow Y_{z_{0}}^{\prime}$ by

$$
\rho_{v}(j)=\phi_{v}^{-1} \alpha_{z}(j) \phi_{v}
$$

where $z=\exp (v) z_{0}$.

The following is straightforward to check:

Lemma 4.1. The deformation $\rho_{v}$ is smooth; that is, the map

$$
B_{\mathfrak{z}}(0, \varepsilon) \times J \times Y \rightarrow Y
$$

given by $(v, h, y) \rightarrow\left(\rho_{v}(j) y\right)$ is smooth. It is also smooth when the actions are restricted to any closed subgroup of $J$.

Remark. We will also denote the action $\rho_{v}$ by $\rho_{z}$ and $\phi_{v}$ by $\phi_{z}$ where $z=\exp (v) z_{0}$. 
We now give an argument to prove Theorem 1.1 in the case where $G$ admits a faithful, irreducible representation. Note that this implies that the center of $G$ is cyclic, and so is not true for all $G$. The general case of Theorem 1.1 will follow from the proof of Theorem 1.2 below. Let $G$ be our simple Lie group, $\Gamma<G$ any lattice and let $\pi: G \rightarrow G L(V)$ be a faithful irreducible representation. Since $G$ is simple and connected, $\pi$ takes values in $S L(V)$. We perform the construction above with $m=\operatorname{dim}(V)$ so that $J=S L(V)=S L(m, \mathbb{R})$ and $H=S L(n, \mathbb{R})$ with $n>m+1$. We describe the centralizer of $G$ or $\Gamma$ in $H$.

Lemma 4.2. The connected component of centralizer of $G$ or $\Gamma$ in $H$ is $D Z$ where $Z$ and $D$ are as above.

Proof. The Lie algebra $\mathfrak{m}$ of the centralizer of $G$ is just the subalgebra of $\mathfrak{h}$ consisting of vectors which are invariant under $\left.\operatorname{Ad}_{H}\right|_{G}$, where $\operatorname{Ad}_{H}$ is the adjoint representation of $H$ into $G L(\mathfrak{h})$. By writing any element of $\mathfrak{h}$ as a block matrix with diagonal blocks of size $n$ and $m$ respectively, it is easy to see that $\left.\operatorname{Ad}_{H}\right|_{G}$ splits as a direct sum of the trivial representation on $\mathfrak{g l}(n-m)$, the restriction $\left.\operatorname{Ad}_{J}\right|_{G}$ on $\mathfrak{s l}(m)$, plus $n-m$ copies of $\pi$ plus $n-m$ copies of the contragradient representation $\pi^{*}$. Since $\pi$ is irreducible, the set of invariant vectors is contained in $\mathfrak{z} \oplus \mathbf{j} \oplus \mathfrak{d}$. All vectors in $\mathfrak{z}$ and $\mathfrak{d}$ are invariant by definition, so it remains to see that there are no invariant vectors in $\mathfrak{j}=\mathfrak{s l}(m)$. The adjoint $\operatorname{Ad}_{J}$ is just the restriction of the conjugation action of $J$ on $\mathfrak{s l}(m)$ and $\mathfrak{s l}(m) \subset \mathfrak{g l}(m)$ which is isomorphic to $V \otimes V^{*}$ as a $J$ module. Now $V \otimes V^{*}$ is isomorphic to $\operatorname{Hom}(V, V)$ as a $J$ or $G$ module, so by Schur's lemma and the fact that $\pi$ is irreducible, the set of $G$ invariant vectors in $V \otimes V^{*}$ is exactly the scalars and there are no $G$ invariant vectors in $\mathfrak{j}$ which is exactly the complement of the scalars.

The centralizer of $\Gamma$ is also an algebraic group whose Lie algebra is the set of $\Gamma$ invariant vectors in $\left.\operatorname{Ad}_{J}\right|_{\Gamma}$. By the Borel density theorem, see e.g. [14, Lemma II.2.3 and Corollary II.4.4], this is the same as the set of $G$ invariant vectors, so we are done.

We now state a more precise version of Theorem 1.1:

Theorem 4.3. Given any $z_{0}$, the $G$ actions on $Y_{z_{0}}$ and $Y_{z}$ are not conjugate as long as $z z_{0}^{-1}$ is small enough. The same is true for the restriction of these actions to $\Gamma$.

Remark. In order to show the existence of an embedded disk in the deformation space, one needs to notice that the bound required on the size of $z z_{0}{ }^{-1}$ is continuous. Therefore by varying $z_{0}$ (as well as $z$ ) we can see that there is a neighborhood of $\rho_{z_{0}}$ where no two actions are conjugate.

Proof. Let $z \in Z$ be small enough so that $z, z z_{0}{ }^{-1} \notin J \Lambda$, and let $\psi: Y \rightarrow Y$ be a diffeomorphism conjugating the action $\rho_{0}$ to the action $\rho_{z}$. By construction, the map $\phi_{z}$ conjugates the action $\rho_{z}$ to the action $\alpha_{z}$. Therefore the map $\phi=\phi_{z}{ }^{-1} \circ \psi$ conjugates the action $\rho_{0}$ to the action $\alpha_{z}$. After deleting the exceptional divisor, the map $\phi$ is clearly an essentially injective, essentially surjective map from $H / \Lambda$ to itself which commutes with the action of $G$ or $\Gamma$. By Corollary 2.1, this implies that $\phi$ is covered by a map of the form $A_{\phi} \circ L_{z_{\phi}}$ almost everywhere, where $z_{\phi}$ is an element of $H$ and $A$ is an automorphism of $H$. Since the only non-trivial automorphism of $H$, conjugate transpose, does not commute with the action of $G$ or $\Gamma$, we know that 
$\phi=L_{z_{\phi}}$ almost everywhere where $z_{\phi}$ is an element of $Z_{H}(G)=Z(H) D Z$. Since $\phi$ is smooth, $\left.\phi\right|_{Y \backslash B}=L_{z_{\phi}}$. We now show that $\phi$ cannot extend to a diffeomorphism of $Y$, obtaining a contradiction.

Since in our first copy of $Y$ we obtain $E$ by blowing up and gluing the orbits $J[e]$ and $J\left[z_{0}\right]$ and in our second copy of $Y$ we obtain $B$ by blowing up and gluing $J[e]$ and $J[z]$, for the map $\phi$ to extend to $H / \Lambda$ would require that $\phi(J[e])=J[e]$ and $\phi\left(J\left[z_{0}\right]\right)=J[z]=z z_{0}{ }^{-1} J\left[z_{0}\right]$ or $\phi(J[e])=J[z]=z J[e]$ and $\phi\left(J\left[z_{0}\right]\right)=J[e]=$ $z_{0}{ }^{-1} J\left[z_{0}\right]$. In either case, $z_{\phi}$ is in $Z(J) Z$ where $Z(J)$ is the center of $J$. This is because $L_{z_{\phi}}$ maps $J[e]$ inside of $J Z[e]$ and therefore $z_{\phi} \in J Z$ by Corollary 3.2. The intersection of $J$ with the centralizer of $G$ must lie in the center of $J$ by Schur's lemma and therefore $z_{\phi} \in Z(J) Z$. In the first case above, we then have that $L_{z_{\phi}} J[e]=J\left[z_{\phi}\right]=J[e]$ and $L_{z_{\phi}} J\left[z_{0}\right]=J\left[z_{\phi} z_{0}\right]=J[z]$. Since $J Z[e]$ is equivariantly a product $J / \Lambda_{J} \times Z / \Lambda_{Z}$, the first equation forces $z_{\phi} \in Z(J) \Lambda_{Z}$, while the second forces $z_{\phi}=z z_{0}{ }^{-1}$ modulo $Z(J) \Lambda_{Z}$, giving a contradiction. The same analysis works in the second case.

Remarks. It is clear from the construction that we have a space of deformations whose dimension is equal to the dimension of $Z$. Since $Z$ can be chosen to be arbitrarily large, this provides examples with arbitrarily large deformation spaces. Similarly, by considering examples where we "blow-up and glue" along $k$ pairs of closed orbits, we can construct actions with deformation spaces of dimension $k \operatorname{dim}(Z)$.

We now turn to the setting of Theorem 1.2 where $G$ is a general connected real algebraic group. Recall from subsection 2.2 that $G=L \ltimes U$ where $U$ is the unipotent radical and $L$ is reductive. By Propositions 2.2 and 2.4, it suffices to consider the case where $L$ is non-compact with compact center. In this case, $L$ is an almost direct product $Z(L) M$ where $Z(L)$ is compact and $M$ is semisimple with at least one non-compact factor. We denote some non-compact adjoint factor of $M$ by $G_{0}$ and note that there is a homomorphism $\sigma: G \rightarrow G_{0}$. We construct an action of $G_{0}$ as above. First choose an irreducible representation of $G_{0}$ which defines a $\operatorname{map} \pi: G_{0} \rightarrow J=S L(m, \mathbb{R})$ for some $m$. We then choose some $n>m+1$ and let $H_{1}=S L(n, \mathbb{R})$. As before the centralizer of $G_{0}$ in $H$ is an algebraic group with connected component $Z=G L(n-m, \mathbb{R})$, and we can construct a cocompact lattice $\Lambda_{1}<H_{1}$ such that $\Lambda_{1} \cap Z=\Lambda_{1}^{Z}$ is a lattice in $Z, \Lambda_{1} \cap J=\Lambda_{1}^{J}$ is a lattice in $J$ and $\Lambda_{1} \cap J Z=\Lambda_{1}^{J Z}$ is a lattice in $J Z$. The construction above gives a family of actions $\rho_{z}^{1}$ of $G_{0}$ (and therefore of $G$ ) on a manifold $Y$ which is obtained from $M_{1}=H_{1} / \Lambda_{1}$ by blowing up two closed $J$ orbits and gluing. Since $G$ is algebraic, we have an embedding of $G$ into $G L(N, \mathbb{R})$ and therefore into $S L(N+1, \mathbb{R})=H_{2}$. We choose an arbitrary cocompact lattice $\Lambda_{2}<H_{2}$. Passing to a subgroup of finite index in $\Lambda_{2}$ so that $Z(G)$ does not intersect $\Lambda_{2}$, there is a faithful $G$ action $\rho^{2}$ on $H_{2} / \Lambda_{2}$ by left translation. Using $\rho_{z}^{1}$ and $\rho^{2}$, we can define a diagonal action of $G$ on $Y \times H_{2} / \Lambda$ which we will denote by $\rho_{z}$. We can now state the following more precise version of Theorem 1.2:

Theorem 4.4. For a given $z_{0}$, the $G$ actions on $\rho_{z_{0}}$ and $\rho_{z}$ are not conjugate as long as $z z_{0}^{-1}$ is small enough. The same is true for the restriction of these actions to any lattice $\Gamma<G$. 
Proof. The proof is analogous to that of Theorem 4.3. The only difference is that applying Corollary 2.1, we can only conclude that $\phi=L_{z_{\phi}}$ where $z_{\phi} \in Z_{H_{1}}\left(G_{0}\right) \times$ $Z_{H_{2}}(G)$. It is straightforward to check that this does not effect our ability to derive a contradiction.

\section{More DEFORMATIONS OF RANK 1 LATtiCE ACTIONS}

In this section, we describe some mixing actions of lattices in $S O(1, n)$ which admit actions on homogeneous spaces for $S O(1, n+1)$ for which there are nontrivial analytic deformations. Note that since lattices in $S O(1, n)$ frequently admit homomorphisms to $\mathbb{Z}$, it is trivial to construct non-faithful actions which admit non-trivial perturbations following the proof of Proposition 2.2. To proceed, we need to recall a construction due to Johnson and Millson. In [12], they construct lattices $\Gamma<S O(1, n)$ which admit continuous families of non-trivial deformations when viewed as subgroups of $S O(1, n+1)$. More precisely:

Theorem 5.1 (Johnson-Millson). There exist both non-cocompact and cocompact lattices $\Gamma<S O(1, n)$ such that

(1) $C=\Gamma \cap S O(1, n-1)$ is a lattice in $S O(1, n-1)$,

(2) there are subgroups $A, B<\Gamma$ such that $\Gamma$ splits as a free product with amalgamation $A *_{C} B$,

(3) let $Z$ be the centralizer in $S O(1, n-1)$ in $S O(1, n+1)$; if $z_{t} \in Z$ is a smooth path, then for $t$ small enough, the group $A *_{C} B^{z_{t}}$ is the image of a discrete faithful homomorphism $\sigma_{t}: \Gamma \rightarrow S O(1, n+1)$ whose image is not conjugate to $\Gamma$ in $S O(1, n+1)$.

This construction is often referred to as bending the lattice. By "bending" along more than one subgroup instead of just $C$, Johnson and Millson construct lattices with large finite dimensional deformation spaces. We leave it to the reader to verify that the construction below produces a deformation space for the group action of the same dimension and only prove the existence of non-trivial deformations.

If we choose a cocompact lattice $\Lambda<S O(1, n+1)$, the homomorphisms $\sigma_{t}$ : $\Gamma \rightarrow S O(1, n+1)$ can be used to define actions $\rho_{t}$ of $\Gamma$ on $S O(1, n+1) / \Lambda$. The theorem of Johnson and Millson can now be interpreted as saying that the actions $\rho_{t}$ are not conjugate in the group of affine diffeomorphisms of $S O(1, n+1) / \Lambda$. (It is easy to see that the group of affine diffeomorphisms is just $S O(1, n+1)$.) We now show that the actions are actually not conjugate in the full diffeomorphism group. Examination of the first proof shows more: the actions are not conjugate by any measurable isomorphism of $S O(1, n+1) / \Lambda$.

Theorem 5.2. The actions $\rho_{t_{1}}$ and $\rho_{t_{2}}$ are conjugate if and only if $t_{1}=t_{2}$. Therefore, for $t$ small enough, $\rho_{t}$ is a non-trivial deformation of $\rho_{0}$.

Remarks. (1) Both the theorem and its proof also apply mutatis mutandi to the other lattices for which Johnson and Millson produce bending deformations. These are lattices $\Gamma$ where there is a non-separating hypersurface rather than a separating hypersurface and so $\Gamma$ can be written as an $H N N$ extension, but not as a free product with amalgamation.

(2) I give two proofs below. As mentioned in the acknowledgements, the second proof given here was discovered jointly with R. Spatzier. 
(3) A third proof of Theorem 5.2, by computation of the entropy of the perturbed actions, was pointed out to the author by Alex Furman. This proof, although it uses much less technology, is somewhat more tedious, as it involves computing entropy for elements not in either $A$ or $B$, and the details are left to the interested reader.

Proof 1: This proof applies only when $n>1$. If $\rho_{t_{1}}$ and $\rho_{t_{2}}$ are conjugate, then there exists $\phi$ in $\operatorname{Diff}(S O(1, n+1) / \Lambda)$ such that $\rho_{t_{1}}(\gamma) \circ \phi=\phi \circ \rho_{t_{2}}$ for any $\gamma \in \Gamma$. Therefore, for any $\gamma \in C$, we have that $\rho_{t_{1}}(\gamma) \circ \phi=\phi \circ \rho_{t_{1}}(\gamma)$ since $\rho_{t_{1}}(\gamma)=\rho_{t_{2}}(\gamma)$ for $\gamma$ in $\Gamma$. Then by Corollary 2.1 and the fact that $\phi$ is small and the automorphism group of $S O(1, n+1)$ is discrete, we have that $\phi=L_{z}$ where $z$ is in $Z=Z_{S O(1, n+1)}(C)=$ $Z_{S O(1, n+1)}(S O(1, n-1))$ since the centralizer of $C$ or $S O(1, n-1)$ in the affine group is contained in the group of translations. Then $\rho_{2}=T_{z^{-1}} \circ \rho_{1} \circ T_{z}$, which implies that $\sigma_{t_{1}}=\sigma_{t_{2}}^{z}$. Applying this equation to any $\gamma \in A$, we see that $\gamma^{z}=\gamma$, which implies that $z$ is trivial since $A$ is Zariski dense by [12, Lemma 5.9]. The same argument applied to $B$ implies that $z_{t_{2}} z=z_{t_{1}}$, a contradiction unless $z_{t_{1}}=z_{t_{2}}$.

Sketch of Proof 2: This proof uses the existence of hyperbolic elements $\gamma_{1}, \ldots, \gamma_{k}$ in $A$ whose common centralizer in $S O(1, n)$ is trivial. We leave it as an exercise for the reader to verify this. Each $\gamma_{i}$ has centralizer $\mathbb{R}_{i} \times S O(n-1)$ in $S O(1, n)$ where $\mathbb{R}_{i}$ is the one parameter group containing $\gamma_{i}$ and centralizer $\mathbb{R}_{i} \times S O(n)$ in $S O(1, n+1)$. The intersection of these centralizers is just a copy of $S O(2)$. We will use the theory of [10] concerning normal hyperbolicity and persistence of central foliations for partially hyperbolic diffeomorphisms. Since the perturbed and unperturbed actions are the same on $A$, we can denote the central foliation for the (un)perturbed action of $\gamma_{i}$ by $W_{i}$. The work of [10] now implies that any conjugacy $\phi$ between the perturbed and unperturbed actions of $\Gamma$ must map each leaf of each foliation $W_{i}$ to itself, since the conjugacy is $C^{0}$ small and the $\gamma_{i}$ are normally hyperbolic with respect to these foliations for both actions. This implies that $\phi$ must map each leaf of the transverse intersection of these foliations to itself. This transverse intersection is just the foliation $\mathfrak{F}$ defined by $S O(2)$ orbits. Therefore $\phi$ can be written as $\phi(x)=z_{x} x$ where $z$ is in $S O(2)$. It is easy to check that the choice of $z_{x}$ is invariant under $C$. By ergodicity of the $C$ action, it then follows that $z_{x}$ is constant, or that $\phi$ is translation by $z$. The proof finishes as before.

Corollary 5.3. There exist affine actions of $S O(1, n)$ on homogeneous spaces of the form $(S O(1, n) \times S O(1, n+1)) /(\Gamma \times \Lambda)$ with non-trivial deformations.

Proof. This follows from Theorem 5.2 and Lemma 2.3. That the induced action is an affine action on $(S O(1, n) \times S O(1, n+1)) /(\Gamma \times \Lambda)$ can be verified using the map

$$
(S O(1, n) \times(S O(1, n+1) / \Lambda) / \Gamma) \rightarrow(S O(1, n) \times S O(1, n+1)) /(\Gamma \times \Lambda)
$$

given by $[g, h]=[g, g h]$ which conjugates the induced action for the unperturbed action to the diagonal action of $S O(1, n)$ on $(S O(1, n) \times S O(1, n+1)) /(\Gamma \times \Lambda)$.

Remark. We note here that although the deformations in Theorem 5.2 are all affine, in Corollary 5.3, only the unperturbed action is affine. More examples of bendings of actions of the groups discussed in this section will be discussed in [5]. 


\section{ACKNOWLEDGEMENTS}

I would like to thank E.J. Benveniste and K. Whyte for many useful conversations concerning the construction and properties of "exotic actions" discussed in this paper. I learned a great deal about these examples during the work on [4] and [9]. I would also like to thank Dave Witte Morris for some useful emails concerning algebraic groups and lattices. A proof that "bending deformations" gave non-trivial deformations of affine actions of lattices and Lie groups was discovered jointly with R. Spatzier during an extremely pleasant visit to the University of Michigan in Spring of 2002. This proof uses the normal form theory of Hirsch-Pugh-Shub for partially hyperbolic diffeomorphisms and is sketched in section 5. That proof led me to the proof using Ratner theory given in section 5, which was the inspiration for the use of Ratner theory in the proofs of Theorem 1.1 and Theorem 1.2. Theorem 1.1 was motivated by a question of $\mathrm{Y}$. Shalom concerning the existence of deformations of actions of lattices in $S U(1, n)$ and $S P(1, n)$.

\section{REFERENCES}

[1] Louis Auslander, On radicals of discrete subgroups of Lie groups. Amer. J. Math. 851963 145-150. MR0152607 (27:2583)

[2] E.J.Benveniste, Exotic Actions of Semisimple Groups and their Deformations, unpublished manuscript and chapter of doctoral dissertation, University of Chicago, 1996.

[3] E.J.Benveniste, Rigidity of isometric lattice actions on compact Riemannian manifolds, GAFA 10 (2000) 516-542. MR1779610 (2001j:37055)

[4] E.J.Benveniste and D.Fisher, Nonexistence of invariant rigid structures and invariant almost rigid structure, Comm. Anal. Geom. 13 (2005) 89-111. MR2154667 (2006f:53056)

[5] D.Fisher, Bending group actions and cohomology of arithmetic groups, in preparation.

[6] D.Fisher and G.A.Margulis, Local rigidity of affine actions of higher rank groups and lattices, preprint.

[7] D.Fisher and G.A.Margulis, Almost isometries, Property (T) and local rigidity, Invent. Math., 162 (2005) 19-80. MR2198325 (2006m:53063)

[8] D.Fisher and G.A.Margulis, Local rigidity for cocycles,in Surv. Diff. Geom. Vol VIII, refereed volume in honor of Calabi, Lawson, Siu and Uhlenbeck, editor: S.T. Yau, 45 pages, 2003. MR2039990 (2004m:22032)

[9] D. Fisher, and K. Whyte, Continuous quotients for lattice actions on compact spaces, Geom. Dedicata, 87 (2001), no. 1-3, 181-189. MR1866848 (2002j:57070)

[10] M.W. Hirsch, C.C. Pugh, and M. Shub, Invariant Manifolds, Lecture Notes in Mathematics 583 , Springer-Verlag, New York, 1977. MR0501173 (58:18595)

[11] T.J.Hitchman, Rigidity theorems for large dynamical systems with hyperbolic behavior. Ph.D. Thesis, University of Michigan, 2003.

[12] Dennis Johnson and John J. Millson, Deformation spaces associated to compact hyperbolic manifolds. Discrete groups in geometry and analysis (New Haven, Conn., 1984), 48-106, Progr. Math., 67, Birkhäuser Boston, Boston, MA, 1987. MR900823 (88j:22010)

[13] A. Katok and J. Lewis, Global rigidity results for lattice actions on tori and new examples of volume-preserving actions. Israel J. Math. 93 (1996), 253-280. MR1380646 (96k:22021)

[14] G.A. Margulis, Discrete subgroups of semisimple Lie groups, Springer-Verlag, New York, 1991. MR1090825 (92h:22021)

[15] S. B. Myers and N. E. Steenrod, The group of isometries of a Riemannian manifold. Ann. of Math. 40 (1939), no. 2, 400-416. MR1503467

[16] Dave Witte Morris, Introduction to Arithmetic Groups, preprint, available at http://people.uleth.ca/ dave.morris/ or via math arxiv.

[17] Richard S. Palais, Equivalence of nearby differentiable actions of a compact group. Bull. Amer. Math. Soc. 67 (1961) 362-364. MR0130321 (24:A185)

[18] V. Platonov and A. Rapinchuk, Algebraic Groups and Number Theory, Academic Press, New York, 1994. MR1278263 (95b:11039) 
[19] M. Ratner, On Raghunathan's measure conjectures, Ann. of Math. 134 no. 3 (1991) 545-607. MR1135878 (93a:22009)

[20] N. Shah, Invariant measures and orbit closures on homogeneous spaces for actions of subgroups generated by unipotent elements, Proceedings of the International Colloquium on Lie Groups and Ergodic Theory Mumbai 1996, edited by S.G. Dani.

[21] D. Witte, Measurable Quotients of Unipotent Translations on Homogeneous Spaces, Trans. Amer. Math. Soc. 354 no. 2 (1994) 577-594. MR1181187 (95a:22005)

[22] D.Witte, Cocycle superrigidity for ergodic actions of non-semisimple Lie groups. Lie groups and ergodic theory (Mumbai, 1996), 367-386, Tata Inst. Fund. Res. Stud. Math., 14, Tata Inst. Fund. Res., Bombay, 1998. MR1699372 (2000i:22008)

[23] R.J. Zimmer, Ergodic Theory and semisimple Groups, Birkhäuser, Boston, 1984. MR776417 (86j:22014)

[24] R.J. Zimmer, Actions of semisimple groups and discrete subgroups. Proc. Internat. Cong. Math. (Berkeley, 1986) 1247-1258 (1987) MR934329 (89j:22024)

[25] R.J. Zimmer, Lattices in semisimple groups and invariant geometric structures on compact manifolds. In: Discrete groups in geometry and analysis, R. Howe, editor. Prog. in Math 67, 152-210. Boston: Birkhauser 1987 MR900826 (88i:22025)

Department of Mathematics, Rawles Hall, Indiana University, Bloomington, Indiana 47405 